\title{
ON THE RANGE OF A HYPONORMAL DERIVATION
}

\author{
JOSEPH G. STAMPF LI ${ }^{1}$
}

ABSTRACT. The inner derivation induced by a hyponormal operat or has closed range if and only if the operator has finite spectrum.

Let $B(H)$ denote the algebra of all bounded linear operators on a Hilbert space $\mathcal{H}$. Define the inner derivation

$$
\Delta_{A}: \mathfrak{B}(\mathcal{H}) \rightarrow \mathfrak{B}(\mathcal{H}) \text { by } \Delta_{A}(X)=A X-X A
$$

for $A, X \in \mathfrak{B}(\mathcal{H})$. For $T \in \mathfrak{B}(\mathcal{H})$ normal, Anderson and Foiaş [1] proved that the range of $\Delta_{T}$ (denoted by $R\left(\Delta_{T}\right)$ ) is norm closed if and only if $a(T)$ is finite. Their proof uses a number of deep results on decomposable operators and asymptotic commutativity. In this note we present a simple proof which enables us to extend their result to hyponormal operators.

The method of proof also permits us to answer partially a question raised by S. R. Caradus. To wit, when is $R\left(\Delta_{T}\right) \cap \mathcal{K}=\Delta_{T}(\mathcal{K})$ where $\mathcal{K}$ is the ideal of compact operators. When $T$ is hyponormal we show that equality holds if and only if $\sigma(T)$ is finite. The following result is a slight variation on a well-known result. See [3, Lemma 2] and subsequent material for example.

Lemm a 1. Let $T \in \mathcal{B}(\mathcal{H})$ be hyponormal. Let $\left\{\lambda_{n}\right\}_{1}^{\infty}$ be a sequence of distinct nonisolated boundary points of $\sigma(T)$. Let $\left\{\epsilon_{n}\right\}_{1}^{\infty}$ be a sequence of positive (nonzero) numbers converging to 0 . Then there exists an orthonormal sequence $\left\{f_{n}\right\}_{1}^{\infty}$ of vectors from $\mathcal{H}$ such that

(1) $\left\|\left(T-\lambda_{n}\right) f_{n}\right\|<\epsilon_{n}$ for $n=1,2, \ldots$, and

(2) $\left(f_{j}, T f_{n}\right)=0$ for $n=1, \ldots, j-1$.

Theorem 1. Let $T \in \mathcal{B}(\mathcal{H})$ be hyponormal. Then $R\left(\Delta_{T}\right)$ is norm closed if and only if $\sigma(T)$ is finite.

Proof. Let $\sigma(T)$ be infinite. Then $\sigma(T)$ has an infinite number of boundary points. We distinguish two cases. If $\sigma(T)$ has an infinite number of isolated boundary points $\left\{\lambda_{n}\right\}_{1}^{\infty}$, then by a well-known result [2] there exists an orthonormal sequence $\left\{f_{n}\right\}_{1}^{\infty}$ such that $T f_{n}=\lambda_{n} f_{n}$ (this case is much easier to handle and the reader may wish to work it out first). If $\sigma(T)$ has an infinite

Received by the editors July 12, 1974.

AMS (MOS) subject classifications (1970). Primary 16A72, 17B05, $18 \mathrm{~B} 20$.

Key words and phrases. Hyponormal operator, inner derivation, closed range, compact operator.

1 The author gratefully acknowledges the support of the National Science Foundation under grant no. GP 29006. 
number of distinct nonisolated boundary points $\left\{\lambda_{n}\right\}_{1}^{\infty}$, we can apply the previous lemma. In this case there exists an orthonormal sequence $\left\{f_{n}\right\}_{1}^{\infty}$ such that $\left\|\left(T-\lambda_{n}\right) f_{n}\right\|<\epsilon_{n}$ and $\left(f_{j}, T f_{n}\right)=0$ for $j>n$. We may further assume the $\lambda_{n}$ 's converge and we choose the $\epsilon_{n}$ 's to satisfy the following conditions:

(1) $\epsilon_{n}>\epsilon_{n+1}>\cdots$;

(2) $\epsilon_{n} \leq\left|\lambda_{n+1}-\lambda_{n}\right|^{2}$ for $n=1,2, \ldots$;

(3) $\sum_{n=1}^{\infty} \epsilon_{n} \eta_{n}<\infty$ where $\eta_{n}=\max _{j=1, \ldots, n}\left|\lambda_{j+1}-\lambda_{j}\right|^{-1 / 2}$.

We set $\mathcal{H}_{1}=\operatorname{clm}\left\{f_{n}\right\}_{1}^{\infty}$ and $\mathcal{H}_{2}=\mathcal{H}_{1}^{\perp}$. If we write $T f_{n}=\mu_{n} f_{n}+\delta_{n}$ where $\left(\delta_{n}, f_{n}\right)=0$ then $\left|\mu_{n}-\lambda_{n}\right|<\epsilon_{n}$ and $\left\|\delta_{n}\right\|<\epsilon_{n}$ for $n=1,2, \ldots$. We will now define operators $V_{m}$ such that $T V_{m}-V_{m} T$ will converge in norm to an operator $A \in \mathfrak{B}(\mathcal{H})$, but $A \neq T W-W T$ for any $W \in \mathfrak{B}(\mathcal{H})$. We define the unbounded operator $V$ as follows: $V f_{n}=\left|\lambda_{n+1}-\lambda_{n}\right|^{-1 / 2} f_{n+1}$ for $n=1,2, \cdots$ and $V g=$ 0 for $g \in \mathcal{H}_{2}$. Let $P_{m}$ be the projection of $\mathcal{H}^{+1}$ onto $\mathbb{N}_{m}=\operatorname{clm}\left\{f_{1}, \ldots, f_{m}\right\}$ and set $V_{m}=V P_{m}$. We claim that $T V_{m}-V_{m} T$ converges in norm to an operator $A \in \mathfrak{B}(\mathcal{H})$. Note first that

$$
\left(T V_{n}-V_{n} T\right) f_{j}=\left\{\begin{array}{lr}
\left|\lambda_{j+1}-\lambda_{j}\right|^{-1 / 2}\left(\mu_{j+1}-\mu_{j}\right) f_{j+1}+\left|\lambda_{j+1}-\lambda_{j}\right|^{-1 / 2} \delta_{j+1}-V_{n} \delta_{j} \\
-V_{n} \delta_{j} \text { for } j>n . & \text { for } j \leq n,
\end{array}\right.
$$

Thus

$$
\left[\Delta_{T}\left(V_{n}\right)-\Delta_{T}\left(V_{m}\right)\right] f_{j}=\left\{\begin{aligned}
& 0 \quad \text { for } j \leq n \leq m, \\
&-\left|\lambda_{j+1}-\lambda_{j}\right|^{-1 / 2}\left(\mu_{j+1}\right.\left.-\mu_{j}\right) f_{j+1}+\left|\lambda_{j+1}-\lambda_{j}\right|^{-1 / 2} \delta_{j+1} \\
&+\left(V_{m}-V_{n}\right) \delta_{j} \quad \text { for } n<j \leq m, \\
&\left(V_{m}-V_{n}\right) \delta_{j} \text { for } n \leq m<j .
\end{aligned}\right.
$$

Note that $\left\|V_{n} \delta_{j}\right\| \leq\left\|V_{n}\right\|\left\|\delta_{j}\right\| \leq n_{n} \epsilon_{j} \leq n_{j} \epsilon_{j}$ for all $n, j$. (The last estimate follows by considering the two cases $n \leq j, n \geq j$ and using the fact that $\left(\delta_{j}, f_{m}\right)=0$ for $m=j+1, j+2, \cdots$ in the latter.) Let $h \in \mathcal{H}_{1}$, and write $h=\sum_{j=1}^{\infty} a_{j} f_{j}$. By a standard argument we see that $\left\|\left[\Delta_{T}\left(V_{n}\right)-\Delta_{T}\left(V_{m}\right)\right\rfloor h\right\|$ $\rightarrow 0$, uniformly in $h$ as $n, m \rightarrow \infty$, since $\left|\lambda_{j+1}-\lambda_{j}\right|^{-1 / 2}\left|\mu_{j+1}-\mu_{j}\right| \rightarrow 0$ as $j \rightarrow \infty$ and $\Sigma \epsilon_{j} \eta_{j}<\infty$. We still must consider vectors $g \in \mathcal{H}_{2}$. For such a $g$,

$$
\left(T V_{n}-V_{n} T\right) g=-V_{n} T g .
$$

Let $T g=\Sigma a_{j} f_{j}+w$ where $w \in \mathcal{H}_{2}$. Then $T^{*} f_{j}=\bar{\mu}_{j} f_{j}+\gamma_{j}$ where $\left(\gamma_{j}, f_{j}\right)=0$. Since $T$ is hyponormal $\left\|\gamma_{j}\right\|<\epsilon_{j}$. Thus $a_{j}=\left(T g, f_{j}\right)=\left(g, T^{*} f_{j}\right)=\left(g, \gamma_{j}\right)$ and hence $\left|a_{j}\right|<\epsilon_{j}$ if $g$ is a unit vector. Hence

$$
\left(T V_{n}-V_{n} T\right) g=-\sum_{j=1}^{n} a_{j}\left|\lambda_{j+1}-\lambda_{j}\right|^{-1 / 2} f_{j+1} .
$$


Finally

$$
\left\|\left(\Delta_{T}\left(V_{n}\right)-\Delta_{T}\left(V_{m}\right)\right) g\right\| \leq \sum_{j=n}^{m}\left|a_{j}\right|\left|\lambda_{j+1}-\lambda_{j}\right|^{-1 / 2} \leq \sum_{n}^{m} \epsilon_{j} \eta_{j}
$$

and the last term tends to zero as $n, m \rightarrow \infty$. Thus $\left\{\Delta_{T}\left(V_{n}\right)\right\}$ is a Cauchy sequence and hence it converges to an operator $A \in \mathfrak{B}(\mathcal{H})$. To complete the first half of the proof we must show that $A \neq T W-W T$. Assume the contrary. Thus $\left((T W-W T) f_{n}, f_{n+1}\right)=\left(A f_{n}, f_{n+1}\right)$ for all $n$ and hence

$$
\begin{aligned}
& \left(\mu_{n+1}-\mu_{n}\right)\left(W f_{n}, f_{n+1}\right)+\left(W f_{n}, \gamma_{n+1}\right)-\left(W \delta_{n}, f_{n+1}\right) \\
& =\left(A f_{n}, f_{n+1}\right)=\left(\mu_{n+1}-\mu_{n}\right)\left|\lambda_{n+1}-\lambda_{n}\right|^{-1 / 2}
\end{aligned}
$$

since $\left(\delta_{n+1}, f_{n+1}\right)$ and $\left(V \delta_{n}, f_{n+1}\right)$ are zero. Thus $\left|\left(W f_{n}, f_{n+1}\right)\right| \geq$ $1 / 2\left|\lambda_{n+1}-\lambda_{n}\right|^{-1 / 2}$ for large $n$ since $\epsilon_{n} /\left|\mu_{n+1}-\mu_{n}\right| \rightarrow 0$. This implies that $W$ is unbounded, contrary to assumption. The other half of the proof will be sketched later.

We now turn to the question of Caradus mentioned in the introduction.

Theorem 2. Let $T \in \mathfrak{B}(\mathcal{H})$ be hyponormal. Then $R\left(\Delta_{T}\right) \cap \mathcal{K}=\Delta_{T}(\mathcal{K})$ if and only if $\sigma(T)$ is finite.

Proof. Again we prove only half the theorem now. Let $\sigma(T)$ be infinite. Proceed as in Theorem 1 and select $\lambda_{n}, f_{n}$ and $\epsilon_{n}$ as before. This time however we define $V f_{n}=f_{n+1}$ for $n=1,2, \cdots$ and $V g=0$ for $g \in \mathcal{H}_{2}$.

By estimates similar to those in Theorem 1 , it is easy to see that $B=$ $T V-V T$ is compact. (Indeed, the operator $A$ in Theorem 1 is compact.) Note that $\left((T V-V T) f_{n}, f_{n+1}\right)=\left(\mu_{n+1}-\mu_{n}\right)$, since the other terms are zero. Assume that $B=T W-W T$ for some noncompact $W \in \mathfrak{B}(\mathcal{H})$. Then

$$
\begin{aligned}
\left((T W-W T) f_{n}, f_{n+1}\right) & =\left(\mu_{n+1}-\mu_{n}\right)\left(W f_{n}, f_{n+1}\right)+\left(W f_{n}, \gamma_{n+1}\right)-\left(W \delta_{n}, f_{n+1}\right) \\
& =\left(B f_{n}, f_{n+1}\right)=\left(\mu_{n+1}-\mu_{n}\right) .
\end{aligned}
$$

Dividing the last equation by $\left(\mu_{n+1}-\mu_{n}\right)$ and letting $n \rightarrow \infty$ we see that $\left(W f_{n}, f_{n+1}\right) \rightarrow 1$. Thus $W$ is not compact and therefore $B \notin \Delta_{T}(\mathcal{K})$.

Remark. Let us now assume that $T$ is hyponormal and $\sigma(T)$ is finite. In that case $T$ must be normal. Thus we write $T=\sum_{j=1}^{n} \lambda_{j} E_{j}$ when the $E_{j}$ 's are just the spectral projections. For $V \in \mathcal{B}(\mathcal{H})$ write $V$ as a matrix $\left[V_{i j}\right]$ on $\mathcal{H}=\sum_{j=1}^{n} \bigoplus E_{j} \mathcal{H}$. Then the $i j$ entry in the matrix representation of $(T V-V T)$ is just $\left(\lambda_{i}-\lambda_{j}\right) V_{i j}$. This observation should make it clear that $R\left(\Delta_{T}\right)$ is closed and moreover that $R\left(\Delta_{T}\right) \cap \mathcal{K}=\Delta_{T}(\mathcal{K})$ since $\left[\left(\lambda_{i}-\lambda_{j}\right) V_{i j}\right]$ is compact if and only if $V_{i j}$ is compact for all $i \neq j$.

Example. In the case of an arbitrary operator $T \in \mathfrak{B}(\mathcal{H})$ we note that 
$\sigma(T)$ finite does not imply $R\left(\Delta_{T}\right) \cap \mathcal{K}=\Delta_{T}(\mathcal{K})$. For example let $T=\left|\begin{array}{ll}0 & Q \\ 0 & 0\end{array}\right|$ on $\mathcal{H} \oplus \mathcal{H}$ where $Q$ is compact. If $R=\left|\begin{array}{ll}A & B \\ C & D\end{array}\right|$ then

$$
T R-R T=\left|\begin{array}{cc}
Q C & Q D-A Q \\
0 & -C Q
\end{array}\right| .
$$

If we set $A=D=0$ and $C=I$ then the operator $\left|\begin{array}{cc}Q & 0 \\ 0 & -Q\end{array}\right|$ is in $R\left(\Delta_{T}\right) \cap \mathcal{K}$. But if $Q$ is a selfadjoint compact operator with trivial kernel then $\left|\begin{array}{cc}Q & 0 \\ 0 & -Q\end{array}\right|$ is clearly not in $\Delta_{T}(\mathcal{K})$.

\section{REFERENCES}

1. Joel H. Anderson and C. Foiaş, On the range of a derivation, Pacific J. Math. (to appear).

2. Joseph G. Stampfli, Hyponormal operators and spectral density, Trans. Amer. Math. Soc. 117 (1965), 469-476; errata, ibid., 550. MR 30 \#3375; 33 \#4686.

3. - Compact perturbations, normal eigenvalues and a problem of Salinas, J. London Math. Soc. (2) 9 (1974), 165-175.

DEPARTMENT OF MATHEMATICS, INDIANA UNIVERSITY, BLOOMINGTON, INDIANA 47401 